\title{
TUATHA DÉ DONANN.
}

Zur Herausgabe dieses Gedichtes von Tanaide (Ĺebor Gabála) benutze ich das Buch von Leinster S. 10, Ballymote S. 33, beide nach Faksimile, ferner Royal Irish Academy D V 1 fol. 9`, Lecan I S. 26 und Ó Cléirigh's Abschrift, herausgegeben von Macalister-MacNeill, Dublin 1916, S.171. Royal Irish Academy und Lecan I besitze ich in der Abschrift, die für mich anzufertigen Herr R. I. Best die grofse Freundlichkeit hatte. Ihm verdanke ich auch die wichtigen Abweichungen in Lecan II (wo Lebor Gabála die Blätter 264 $\mathrm{r}-302^{\mathrm{v}}$ einnimmt).

Lebor Gabála in Leinster gehört zur Fassung $\mathrm{A}$ in Lecan I, und Royal Irish Academy zur verwandten B I. B III enthält Tuatha Dé Donann fo diamair noch nicht. In Royal Irish Academy, sonst einer der besten Handschriften, ist unser Gedicht häufig überschrieben, oft in einer Weise, die von der ersten Schrift abweicht. Den ältesten Fassungen A und B unseres Gedichtes steht die jüngere $\mathrm{C}$ gegenüber, durch Ballymote und Lecan II vertreten. Noch jünger ist Ó Cléirigh, der u. a. Leinster benutzte, während sonst unsere Handschriften als voneinander unabhängig gelten dürfen.

Ich weiche nie ganz von der Überlieferung $a b$ und benutze C nur ausnahmsweise. In der. Schreibweise und im rein Sprachlichen ziehe ich Älteres vor, sonst wähle ich die Textform, die $A$ und B I gemeinsam ist, und lasse in Zweifelsfällen $C$ den Ausschlag geben, solange sich nicht aus dem Reim die richtige Lesung von selbst ergibt. Ó Cléirigh benutze ich bei der Herstellung des Textes im allgemeinen nicht. 
Der Dichter heifst Leinster $10 \mathrm{~b}$ : Tanaidhe; ${ }^{1)}$ Ballymote 18 a 2: Tanaidhe $0^{2}$ Dubhsailech; Ó Cléirigh (bei MacalisterMac Neill S. 172 § 120), oder vielmehr der Zusatz einer späteren Hand in seinem Werke nennt ihn Tanaidhe O'Maol Conaire. O'Reilly, Irish Writers p.LXXXII f. gibt Tanaidhe O'Mulconaire $\dagger 1136$ als Verfasser unseres Gedichtes und Firbolg batar sunda seal (vgl. Ó Cl. Macalister-MacNeill S. 136, wo wieder eine spätere Hand den Namen nachtrug). Best (brieflich) bezeichnet den Namen Ó Dubhshailech als Spottnamen" (des Geschlechtes). Er findet Annála Locha Cé zum Jahre 1270 und vier Meister 1270 einen Streit zwischen Dubhshailech O'Maelchonaire und Tanaide Mór O'Maelchonaire um die Würde des Ollamh erwähnt.

\section{Text.}

a Tuatha Dé Donann ${ }^{1}$ fo diamair fir ${ }^{2}$ cen chomall crābuid cuileoin in chaîlli na chrínaigh ${ }^{3}$ daīni dfuilfeóil Ādaim.

b Uaisli thall ${ }^{1}$ na tuaithe tréine lucht na cruaiche críne aisnēidim $^{2}$ sin trēimsi ị̂támni ${ }^{3}$ a rēimse sa ríge.

\section{Übersetzung.}

a Die Stämme der Göttin Donu unter Geheimnis, Männer ohne Erfüllung der Andacht, SpröIslinge (wörtlich: junge Hunde) des Landes, das nicht welkte, Männer vom Blutfleische Adams.

b Edle drüben vom starken Stamme, die Leute vom verwitterten Hügel. Ich verkünde zur Zeit, da wir leben, ihre Dauer und ihr Reich.

\section{Varianten.}

$a=$ Leinster $; \quad b=\operatorname{Lecan} I(B I) ; \quad R=$ Royal Irish Academy $D V 1 ;$ $L=$ Lecan II (wo nichts angegeben, $L=b$ ); $B=$ Ballymote; $C=O$ OCléirigh

a) $1 \quad a B$ : danann $2 b$ (nicht $L) R C$ : lucht $3 \quad b R$ : rot chrinaig

b) $1 C$ : tall $2 a$ : aisneidem; $b R$ : imraidim; $L B$ : aisneidfead; $C$ : iomraidem $3 C$ : ataimni

1) Vgl. Meyer, Primer of Irish Metrics, Index of Poets, wo LI, 106 für LL $10 \mathrm{~b}$ steht. 
c Ré ${ }^{1}$ secht mbliadna ${ }^{2}$ Nuadait $^{3}$ nársing ${ }^{4} o_{\text {ossin }}^{5}$ chuanairt chéibfinn ${ }^{6}$

flaithius ind ${ }^{7}$ fir chīcair ${ }^{8}$ chūlfind $^{9}$ ria $^{10}$ tĩochtain $^{11}$ i $\mathrm{n} \overline{\mathrm{E}}$ rinn. 12

d I mmaig ${ }^{1}$ Tuired truim ${ }^{2}$ cen $^{3}$ trucha ${ }^{4}{ }^{-}$torchair $^{5}$ cuing chatha

do chosnamaid bán in betha ${ }^{6} \mathrm{ro}^{\circ}$ leodad $^{7}$ lám $^{8}$ f́latha.

e Secht mbliadna Breissi nī bánfatat ${ }^{1}$ tria $^{2}$ deissi don duanabb ${ }^{3}$ i flaithius ${ }^{4}$ ósin $^{5}$ chlár chnóbocc cor ${ }^{-1}$ ccad ${ }^{6}$ lám Nuadat.

c Eine Zeit von sieben Jahren war die des Nuadhu über die haarblonde Meute, die Herrschaft des beutegierigen Mannes im hellen Haar, bevor sie nach Island kamen.

d Auf dem Tuiredfelde schwier, dem unvergänglichen, wo ein Schlachtenheld fiel, wurde dem weifsen Streiter der Welt eine Fürstenhand abgehauen.

e Sieben Jahre herrschte Breise - es war kein glänzender Zeitraum durch Nutzen für den Dichter (wörtlich: Liederabt. B. ist sehr karg gegen die Dichter, wie den Cairbre mac Étáine, vgl. Stokes, The second Battle of Moytura, Revue Celtique 12 S. 71) - über die Ebene mit den weichen Nüssen, bis die Hand des Nuadhu geheilt wurde.

c) 1 fehlt bei b(nicht L) $R C$ : 2 so ausgeschrieben bei $B$ (vielleicht als Acc. Pl. nach Präp. re) 3 aLB: nuadat; $b R$ : do nuadait $4 a$ : nareng; $b R$ : naršeng; $C$ : nársing $5 \cdot B$ : uasan; $C$ : os an $6 b R$ : ceilbinn; $L$ : ceolbinn; $B$ : ceblfind; $C$ : ccuanairt ccélbiun $7 C:$ an $8 a$ : chichmair; $B$ : chichair $9 b R B C$ : chuilfind $10 C$ : re $11 a b R E$.: tiachtain. C's Verbessérung durch Rein gefordert. Urtext jedenfalls: tīchtain $12 \quad b R B C:$ a

d) 1 bRBC: muigh; $L$ : ar muigh $2 \cdot b$ : thruim $3 L$ : cin; $C$ : co $4 L$ : triucha $5, b$ : car fargbadh; $R$ : ca fargbadh; $L$ : adrochair; $B$ : adrochaing; $C$ : go ffarcaib $6 \mathrm{~B}$ : bain mbetha 7 a: ro lead; $b R C$ : ro leod; $L$ : do leonad; $B$ : re leonam $8 b($ nicht $L) R C$ : a lam. Das wäre nach do chosnamaid zu erwarten, wenn eine andere Form als-leonad denkbar wäre. Doch lassen $a$ und $B$ a fort.

- e) 1 a: na mbánat; $L:$ ni buanat; $C:$ nir bhanadh; $C:$ nir bánfad. duanabb kann'schwerlich, wie a anzunehmen scheint, den die Dichter vernachlässigenden Breise bedeuten. An die Dichter selbst ist gedacht, besonders wohl an Cairpre mac Etaine, vgl. Second Battle of Mag Tuired: Whitley Stokes Nr. 39. Revue Celtique S. 70. 2 a: lór a ; LB: andeise $3 L$ : duanbog $4 R$ : flaithsi $5 b$ (nicht $L) R C$ : forin $6 a$ : coro iccad; $B$ : gur coirgeadh 
f Nuádo iar $\sin ^{1}$ fiche bliadan co Lùg rindāgach do rīgad ${ }^{4}$

rue sithi ${ }^{2}$ for sluagad ${ }^{3}$ ildanach ${ }^{5}$ cen $^{6}$ uarad. 7

g. Cethracha do Lug ba ${ }^{1}$ lomda2 i rrigi ${ }^{3} \overline{0 s}^{4}$ brug Banba. nírānaic nochtcholba nemda ${ }^{5}$. ochtmoga don ${ }^{6}$ Dagda.

h Deich mbliadna don ${ }^{1}$ Delbæth dichra co ricc $^{2}$ remgæth riachda ${ }^{3}$

cen cleith ${ }^{4} \overline{0} \mathrm{~s}_{\text {braine }}{ }^{5}$ na bōchna ${ }^{6}$ a deich aile dFíachna.

i Noí mbliadna fichet fōs fegaim ${ }^{2} \quad \overline{0}$ cach $^{3}$ sithert ${ }^{4}$ dĒrinn ${ }^{5}$ i rrìgi for Banba bānuill ${ }^{6}$ duīb ${ }^{7}$ in Dagda dēinseing. ${ }^{8}$

f Nuadhu darauf zwanzig Jahre machte Stürme auf der Heeresfahrt, bis Lugh, der Speerkämpfer, zum Könige gemacht wurde, der kunstreiche, der nie erlahmte (wörtlich: ohne zu erkalten).

g. Vierzig Jahre dem Lugh, er war gleichwertig, im Königtum über das Land von Banbha - er erreichte kein himmlisches Nachtlager (wörtlich: Nacht-Bettpfosten, d. h. die ewige Ruhe. Der Gedanke, dafs hier, wie in nochtadaig - O'Dav. - nocht = Nacht in seiner ursprünglichen substantivischen Bedeutung steht, scheint weniger gekünstelt als der Versuch, nocht = nackt vorauszusetzen).

h Zehn Jahre dem hitzigen Dealbhaeth, bis eine vorzeitige qualvolle Wunde (seine Ermordung durch Fiachna) ohne Hehl über den Herrn der See kam, weitere zehn dem Fiachna.

i Neunundzwanzig Jahre seh ich noch über jedes weite Gebiet von Irland im.Königtum über Banbha, strahlend (wörtlich: weifs) und grofs, die Enkel des heftigen, schlanken Daghdha.

f) $1 a$ : sein $2 a$ : saide; $R:$ sith; $B:$ sidhi $3 L B$ : sluaghaib $4 a$ : co lug dia crigad $5 R$ : illdhanach; $a B$ : illanach $6 B C$ : gan $7 B$ : fhuarad; $C$ : fuarad

g) $1 L$ : fa $2 L B$ : learrda $3 L B$ : na righi $4 b$ (nicht $L) R$ : ar $5 L$ neamda $\bar{s}$ ochtmoga $6 L$ : ind

h) $1 L B$ : do $2 b$ : cosric; $a B$ : co rrig; $L$ : rich; $C$ : gus rice $3 L$ : riachna; $B$ : riachta $4 a$ : locht $5 b$ : bruine; $B$ : bruinde; $C$ : broine $6 a C:$ mbxthga; $B: \mathrm{m}[$ boch]na

i) 1 bRC: deich $2 a$ : fogart; $B$ : fegham; $C$ : fosfiadaim $3 a$ : os chach; $b$ (nicht $L)$ : nasgach; $R$ : uas cach; $C$ : os'cech $4 a B$ : fithirt; $b$ : sithbert; $R$ : sithfeirt; $C$ : sithfert 5 a: dering 6 bRC: brnigbinn; $L B$ : buanuaill $7 B$ : duail $8 a$ : for herind; $b$ RRB : densheng; $C$ : deinsing 
j Andsin ${ }^{1}$ tāncatar mic ${ }^{2}$ Mīled rāncatar $^{3}$ dia $^{4}$ ruadad $^{5}$ cland in merscáil móir ${ }^{6}$ ro $^{\circ}$ maíded $^{7}$ a hEspāin cen uarad.

k Coros gonsat ${ }^{1}$ Gæidil gnithe cen $^{2}$ toīdin tria thuaichli ní do braissi ni do baithi bec taissi na tuaithe.

j Da kamen die Söhǹe des Míl, sie kamen, sie zu röten, die Kinder des grolsen Riesen, rühmte man, aus Spanien ohne zu erlahmen.

k Und die tatenreichen Gälen töteten sie ohne (grosse) Schar durch List. Es ist keine leichtsinnige noch einfältige Behauptung: klein sind die Reste des Stammes.

j) $1 \mathrm{~L}: \operatorname{arsin} ; B C$ : iar sin $2 C:$ meic $3 L$ : dorancatar $4 L$ : co $5^{\prime} a$ : radath; $b R$ : ruamnad; $L:$ ruadag; $C$ : ruamadh $6 b R$ : cland mor in merscail moir; $L$ : clanda in merscail moir. $7 L$ : cen maidem; $B$ : re maidhem

k) $1 L B$ : gorgabsat $2 a B C$ : con

Bonn.

Gustav Lehmacher, S. J. 